MITSUBISHI ELECTRIC RESEARCH LABORATORIES

http://www.merl.com

\title{
Extremum Seeking-based Adaptive Control for Electromagnetic Actuators
}

\author{
Benosman, M.; Atinc, G.M.
}

TR2014-090 September 2014

\begin{abstract}
In this paper we present a learning-based adaptive method to solve the problem of robust trajectory tracking for electromagnetic actuators. We merge a nonlinear backstepping controller that ensures bounded input/bounded states stability, with a multi-variable extremum seeking (MES) model-free learning algorithm. The learning algorithm is used to estimate online the uncertain parameters of the model, in this sense we propose a learning-based adaptive controller. We present a proof of stability of this learning-based nonlinear controller when considering uncertainties with linear parametrization. The efficiency of this approach is shown on a numerical example.
\end{abstract}

International Journal of Control

This work may not be copied or reproduced in whole or in part for any commercial purpose. Permission to copy in whole or in part without payment of fee is granted for nonprofit educational and research purposes provided that all such whole or partial copies include the following: a notice that such copying is by permission of Mitsubishi Electric Research Laboratories, Inc.; an acknowledgment of the authors and individual contributions to the work; and all applicable portions of the copyright notice. Copying, reproduction, or republishing for any other purpose shall require a license with payment of fee to Mitsubishi Electric Research Laboratories, Inc. All rights reserved. 



\title{
RESEARCH ARTICLE
}

\section{Extremum Seeking-based Adaptive Control for Electromagnetic Actuators}

\author{
Mouhacine Benosman ${ }^{a}$ and Gökhan M. Atınç ${ }^{b}$ \\ (Received 00 Month 200x; final version received 00 Month 200x)
}

\begin{abstract}
In this paper we present a learning-based adaptive method to solve the problem of robust trajectory tracking for electromagnetic actuators. We merge a nonlinear backstepping controller that ensures bounded input/bounded states stability, with a multi-variable extremum seeking (MES) model-free learning algorithm. The learning algorithm is used to estimate online the uncertain parameters of the model, in this sense we propose a learningbased adaptive controller. We present a proof of stability of this learning-based nonlinear controller when considering uncertainties with linear parametrization. The efficiency of this approach is shown on a numerical example.
\end{abstract}

Keywords: learning control, adaptive control, nonlinear control, electromagnetic actuators.

\section{Introduction}

Electromagnetic actuators are used in many different systems, such us in combustion engines, opening and closing cargo doors in aircraft systems, precision positioning stages actuation, etc. This work deals with a particular control problem of nonlinear electromagnetic actuator, namely, the robust 'soft landing' problem, which requires accurate control of the moving element of the actuator between two desired positions. Soft landing aims at achieving small contact velocities, thus reducing the noise of the actuator and ensuring low component wear of the actuator. Furthermore, the soft landing property of an actuator has to be guaranteed over long periods of time during which the actuator's components may age slowly. Due to these practical constraints we have developed a robust control algorithm that aims for a zero impact velocity, and adapts to the system aging via a learning-based adaptive algorithm. We present here the results of this study.

Many papers have been dedicated to the soft-landing problem for electromagnetic actuators,

${ }^{a}$ Mitsubishi Electric Research Laboratories, 201 Broadway Street, Cambridge, MA 02139, USA ( Corresponding author. Email: benosman@merl.com)

${ }^{b}$ Mechanical Science and Engineering Department and the Coordinated Science Laboratory, University of Illinois at UrbanaChampaign, USA 
e.g. Hoffmann (2003), Tsai (2008), Peterson and Stefanopoulou (2004), Tai and Tsao (2002), Peterson and Stefanopoulou (2003), Eyabi and Washington (2006), Kahveci and Kolmanovsky (2010), Benosman and Atinc (2013a,b). Some linear controllers have been proposed in Hoffmann (2003), Tai and Tsao (2002). The results based on linear control theory use linearized models of the actuator dynamics and thus are usually designed to operate in a small neighborhood of the linearization points. To control the system over a larger operation space, the controller has to be based on more complex nonlinear models of the actuators. Thus, in this paper we consider the nonlinear dynamics of the system for the control design. Various nonlinear controllers have been used in Tsai (2008), Peterson and Stefanopoulou (2004, 2003), Eyabi and Washington (2006), Kahveci and Kolmanovsky (2010), Benosman and Atinc (2013a,b). In Tsai (2008), the authors studied the problem of electromagnetic valve actuator control in an internal combustion engine. The proposed solution is based on iteratively solving a constrained nonlinear optimal problem using Nelder-Mead algorithm. The robustness of this approach to system's aging was not shown, and there were no feedback terms to robustify the feedforward control. In Peterson and Stefanopoulou (2003), the authors proposed a nonlinear control based on Sontag's feedback to solve the problem of armature stabilization for an electromechanical valve actuator. However, this approach did not solve the problem of armature trajectory tracking and did not consider robustness of the controller with respect to system's uncertainties. In Kahveci and Kolmanovsky (2010), the authors designed a backstepping-based controller for the electromagnetic actuator, but uncertainties in the parameters of the system were not considered in this paper. In Eyabi and Washington (2006), a nonlinear sliding mode approach was used to solve the problem of trajectory tracking for an electromagnetic valve actuator. The reported results showed good tracking performances, however, the robustness with respect to uncertainty in the system parameters was not guaranteed. In Peterson and Stefanopoulou (2004), the authors used a single parameter extremum seeking learning method along with a nonlinear controller to solve the problem of armature trajectory tracking for an electromechanical valve actuator. Although the learning algorithm was not directly tailored to ensure robustness of the controller to model uncertainties or parameters drift over time, this robustness was intrinsic due to the iterative nature of the learning process. However, in this controller only a scalar gain of the control was tuned online, and there was no explicit proof of robustness of the controller with respect to model uncertainties. In Benosman and Atinc (2013a), the authors proposed a robust controller for nonlinear electromagnetic actuators, which was based on Lyapunov redesign techniques. The proposed nonlinear controller was complemented by a multi-variable extremum seeking control to tune the feedback gains in order to improve the control robustness w.r.t model uncertainties. Some preliminary results related to the present work were presented in Benosman and Atinc 
(2013b), where the authors designed a backstepping-based controller for electromagnetic actuators which was robustified by an extremum seeking algorithm to estimate uncertain parameters of the system, however, no rigorous analysis was present concerning the stability of the combined model-based nominal controller and the model-free learning algorithm. The proof of stability was subsequently introduced by the same authors in Atinc and Benosman (2013). In Benosman and Atinc (2013), the authors used classical adaptive control, i.e., indirect model-based adaptive control, to solve the problem of robust soft-landing for electromagnetic systems. The method relied on a nonlinear controller merged with a nonlinear model-based gradient descent filters to estimate the uncertain parameters of the model. The controllers showed robust performances w.r.t. model uncertainties, however, this approach could not deal with more than one uncertainty at a time, due to some fundamental limitations of the gradient-descent model-based adaptive approach (Benosman and Atinc (2013)).

In this work, we use a nonlinear model of the electromagnetic actuator to design a nonlinear backstepping controller that ensures integral Input to State stability (iISS) between the tracking error state and the uncertain parameters estimation error input. Subsequently, the controller is robustified by a model-free MES algorithm which is used to identify online some uncertain parameters of the model; this includes tracking over time any slow drifts of these parameters that might occur due to the system aging. Notice that contrary to Peterson and Stefanopoulou (2004), Benosman and Atinc (2013a), we are using a MES approach to learn a vector of the model's parameters, and not the gain of the controller. In this sense, we are proposing a learning-based adaptive control. Furthermore, we present here the stability analysis of the whole controller; i.e., the nominal controller merged with the MES learning algorithm, for the case of model uncertainties with linear parametrization.

This paper is organized as follows: We first recall some useful definitions in Section 2. Next, a nonlinear model of electromagnetic actuators is presented in Section 3. Section 4 is dedicated to the main result of this work, namely, the learning-based adaptive nonlinear controller design and stability analysis. Numerical validation of the proposed controller is given in Section 5, and concluding remarks are stated in Section 6.

\section{Preliminaries}

Throughout the paper we will use $\|$.$\| to denote the Euclidean norm, i.e., for x \in \mathbb{R}^{n}$ we have $\|x\|=\sqrt{x^{T} x}$. We will use the notations $\operatorname{diag}\left\{m_{1}, \ldots, m_{n}\right\}$ for $n \times n$ diagonal matrix, and (.) for the short notation of time derivative. We denote by $C^{k}$ functions that are $k$ times differentiable. A function is said to be analytic in a given set, if it admits a convergent Taylor series approximation 
in some neighborhood of every point of the set. A continuous function $\alpha:[0, a) \rightarrow[0, \infty)$ is said to belong to class $\mathcal{K}$ if it is strictly increasing and $\alpha(0)=0$. A continuous function $\beta:[0, a) \times[0, \infty) \rightarrow[0, \infty)$ is said to belong to class $\mathcal{K} \mathcal{L}$ if, for each fixed $s$, the mapping $\beta(r, s)$ belongs to class $\mathcal{K}$ with respect to $r$ and, for each fixed $r$, the mapping $\beta(r, s)$ is decreasing with respect to $s$ and $\beta(r, s) \rightarrow 0$ as $s \rightarrow \infty$.

Let us now introduce some definitions that will be used subsequently.

Definition 2.1: [Local Integral Input-to-State Stability (Ito and Jiang (2009))] Consider the system

$$
\dot{x}=f(t, x, u)
$$

where $x \in \mathcal{D} \subseteq \mathbb{R}^{n}$ (D compact) such that $0 \in \mathcal{D}$, and $f:[0, \infty) \times \mathcal{D} \times \mathcal{D}_{u} \rightarrow \mathbb{R}^{n}$ is piecewise continuous in $t$ and locally Lipschitz in $x$ and $u$, uniformly in $t$. The inputs are assumed to be measurable and locally bounded functions $u: \mathbb{R}_{\geq 0} \rightarrow \mathcal{D}_{u} \subseteq \mathbb{R}^{m}$ ( $\mathcal{D}_{u}$ compact). Given any control $u \in \mathcal{D}_{u}$ and any $\xi \in \mathcal{D}_{0} \subseteq \mathcal{D}$, there is a unique maximal solution of the initial value problem $\dot{x}=f(t, x, u), x\left(t_{0}\right)=\xi$. Without loss of generality, assume $t_{0}=0$. The unique solution is defined on some maximal open interval, and it is denoted by $x(\cdot, \xi, u)$. System (1) is locally integral input-to-state stable (LiISS) if there exist functions $\alpha$, $\gamma \in \mathcal{K}$ and $\beta \in \mathcal{K} \mathcal{L}$ such that, for all $\xi \in \mathcal{D}_{0}$ and all $u \in \mathcal{D}_{u}$, the solution $x(t, \xi, u)$ is defined for all $t \geq 0$ and

$$
\alpha(\|x(t, \xi, u)\|) \leq \beta(\|\xi\|, t)+\int_{0}^{t} \gamma(\|u(s)\|) d s
$$

for all $t \geq 0$. Equivalently, system (1) is LiISS if and only if there exist functions $\beta \in \mathcal{K} \mathcal{L}$ and $\gamma_{1}, \gamma_{2} \in \mathcal{K}$ such that

$$
\|x(t, \xi, u)\| \leq \beta(\|\xi\|, t)+\gamma_{1}\left(\int_{0}^{t} \gamma_{2}(\|u(s)\|) d s\right)
$$

for all $t \geq 0$, all $\xi \in \mathcal{D}_{0}$ and all $u \in \mathcal{D}_{u}$. Note that if system (1) is LiISS, then the 0 -input system is locally uniformly asymptotically stable (0-LUAS), that is, the unforced system

$$
\dot{x}=f(t, x, 0)
$$

is LUAS (Sontag and Wang (1996)).

Definition 2.2: [iISS-Lyapunov (Ito and Jiang (2009), Angeli (2000))] A $C^{1}$ function $V$ : $[0, \infty) \times \mathcal{D} \rightarrow \mathbb{R}$ is called an iISS-Lyapunov function for system (1) if there exist functions $\alpha_{1}$, $\alpha_{2}, \sigma \in \mathcal{K}$, and a continuous positive definite function $\alpha_{3}$, such that

$$
\alpha_{1}(\|x\|) \leq V(t, x) \leq \alpha_{2}(\|x\|)
$$

for all $x \in \mathcal{D}$ and

$$
\dot{V} \leq-\alpha_{3}(\|x\|)+\sigma(\|u\|)
$$

for all $x \in \mathcal{D}$ and all $u \in \mathcal{D}_{u}$. 
Definition 2.3: [Weakly Zero-Detectability (Angeli (2000)) ] Let an output for the system (1) be a continuous map $h: \mathcal{D} \rightarrow \mathbb{R}^{p}$, with $h(0)=0$. For each initial state $\xi \in \mathcal{D}_{0}$, and each input $u \in \mathcal{D}_{u}$, let $y(t, \xi, u)$ be the corresponding output function; i.e., $y(t, \xi, u)=h(x(t, \xi, u))$, defined on some maximal interval $\left[0, T_{\xi, u}\right)$. The system (1) with output $h$ is said to be weakly zero-detectable if, for each $\xi$ such that $T_{\xi, 0}=\infty$ and $y(t, \xi, 0) \equiv 0$, it must be the case that $x(t, \xi, 0) \rightarrow 0$ as $t \rightarrow \infty$.

Definition 2.4: $\quad$ [Smooth Dissipativity (Angeli (2000))] System (1) with output $h$ is dissipative if there exists a $C^{1}$, proper and positive definite function $V$, together with a $\sigma \in \mathcal{K}$ and a continuous positive definite function $\alpha_{4}$, such that

$$
\dot{V} \leq-\alpha_{4}(\|h(x(t, \xi, u))\|)+\sigma(\|u\|)
$$

for all $x \in \mathcal{D}$ and all $u \in \mathcal{D}_{u}$. If this property holds with a $V$ that is also smooth, system (1) with output $h$ is said to be smoothly dissipative. Finally, if (7) holds with $h=0$, i.e., there exists a smooth proper and positive definite $V$, and a $\sigma \in \mathcal{K}$, so that

$$
\dot{V} \leq \sigma(\|u\|)
$$

holds for all $x \in \mathcal{D}$ and all $u \in \mathcal{D}_{u}$, the system (1) is said to be zero-output smoothly dissipative.

\section{System modelling}

We recall below a nonlinear model of the electromagnetic actuator presented in Peterson and Stefanopoulou (2004):

$$
\begin{aligned}
& m \frac{d^{2} x}{d t^{2}}=k\left(x_{0}-x\right)-\eta \frac{d x}{d t}-\frac{a i^{2}}{2(b+x)^{2}}+f_{d} \\
& u=R i+\frac{a}{b+x} \frac{d i}{d t}-\frac{a i}{(b+x)^{2}} \frac{d x}{d t}, 0 \leq x \leq x_{f}
\end{aligned}
$$

where, $x$ represents the armature position mechanically constrained between the initial position of the armature 0 , and the maximal position of the armature $x_{f}, \frac{d x}{d t}$ represents the armature velocity, $m$ is the armature mass, $k$ the spring constant, $x_{0}$ the initial spring length, $\eta$ the damping coefficient, $\frac{a i^{2}}{2(b+x)^{2}}$ represents the electromagnetic force (EMF) generated by the coil, $a, b$ being constant parameters of the coil, $f_{d}$ a constant term modelling unknown disturbance force, e.g. static friction, $R$ the resistance of the coil, $L=\frac{a}{b+x}$ the coil inductance (assumed to be dependent on the position of the armature), $\frac{a i}{(b+x)^{2}} \frac{d x}{d t}$ represents the back EMF, $i$ denotes the coil current, $\frac{d i}{d t}$ its time derivative and $u$ represents the control voltage applied to the coil. In the next section, based on this nonlinear model of the electromagnetic actuator, we first 
develop a backstepping nonlinear controller and then we extend it to its adaptive version using a MES algorithm.

\section{Learning-based adaptive nonlinear control}

\subsection{Backstepping Controller with Guaranteed Integral Input-to-State Stability}

In this section, we will first state a result discussed in Angeli (2000) for autonomous systems, and then prove that the sufficiency part of these results also hold for non-autonomous systems. Subsequently, we will make use of these results to discuss the stability of the backstepping merged with the MES algorithm.

Theorem 4.1: [Equivalent Characterizations of iISS (Angeli (2000))] Consider the autonomous system

$$
\dot{x}=f(x, u)
$$

where $x \in \mathbb{R}^{n}, f: \mathbb{R}^{n} \times \mathbb{R}^{m} \rightarrow \mathbb{R}^{n}$ is locally Lipschitz and the inputs are measurable and locally bounded functions $u: \mathbb{R}_{\geq 0} \rightarrow \mathbb{R}^{m}$. The unique solution of the initial value problem $\dot{x}=f(x, u)$ with $x(0)=\xi$ defined on some maximal open interval is denoted by $x(\cdot, \xi, u)$. The following properties are equivalent for the system (10): 1) The system is iISS. 2) The system admits a smooth iISS-Lyapunov function. 3) There exists an output that makes the system smoothly dissipative and weakly zero-detectable. 4) The system is 0-globally asymptotically stable (0-GAS) and zero-output smoothly dissipative.

Remark 1: Note that we will analyze the local stability properties of the electromagnetic actuator system, hence we do not require conditions that give global iISS properties. To this purpose, we will modify the 0-GAS condition to 0-LUAS for the non-autonomous system. Moreover, we only need sufficiency, hence smoothness condition of iISS Lyapunov functions which was used in Angeli (2000) to prove necessity, is not required here. Thus, we modify properties $1-4$ of Theorem 4.1 to the following ones for the non-autonomous system (1): [1a.] The system is LiISS. [2a.] The system admits a continuously differentiable iISS-Lyapunov function. [3a.] There exists an output that makes the system dissipative and weakly zero-detectable locally. [4a.] The system is 0-LUAS and zero-output dissipative.

Now we propose the following lemma.

Lemma 4.2: $\quad$ Consider the non-autonomous system (1). If there exists an output that makes the system dissipative and weakly zero-detectable locally, then the system is LiISS. 


\section{Proof:}

If we interpret Lemma 4.2 in terms of the modified conditions of Remark 4.1, then Lemma 4.2 states that for non-autonomous systems, if $3 a$ holds, then $1 a$ is true. To prove this lemma, we will first show that $3 a \Longrightarrow 4 a$; then, we will show $4 a \Longrightarrow 2 a$, and finally we will prove that $2 a \Longrightarrow 1 a$.

$\underline{3 a \Longrightarrow 4 a}$ : Assume that some output $h(\cdot)$ that makes the system weakly-zero detectable locally, and there exist a $C^{1}$ proper positive definite function $V$, a function $\sigma \in \mathcal{K}$ and a continuous positive definite function $\alpha_{4}$ such that

$$
\dot{V} \leq-\alpha_{4}(\|h(x)\|)+\sigma(\|u\|)
$$

hold for all $x \in \mathcal{D}$ and all $u \in \mathcal{D}_{u}$. With $u=0$, we have $\dot{V} \leq-\alpha_{4}(\|h(\xi)\|)$, and since the system is weakly-zero detectable, by LaSalle-Yoshizawa Theorem (Haddad and Chellaboina (2008)), we conclude that the system (10) is 0-LUAS. Also, since $-\alpha_{4}(\|h(x)\|) \leq 0$, we have $\dot{V} \leq \sigma(\|u\|)$ from (11), implying, by Definition 2.4, that the system is zero-output dissipative.

$\underline{4 a \Longrightarrow 2 a}$ : Assume $4 a$ holds. Since the system is 0-LUAS, by a converse Lyapunov theorem (e.g., Haddad and Chellaboina (2008)), there exists a $C^{1}$ function $V_{0}$ for the system (1) such that

$$
\begin{gathered}
\alpha_{1}(x) \leq V_{0}(t, x) \leq \alpha_{2}(x) \\
\frac{\partial V_{0}}{\partial t}+\frac{\partial V_{0}}{\partial x} f(t, x, 0) \leq-\alpha_{0}(\|x\|), \quad \forall x \in \mathcal{D}
\end{gathered}
$$

holds for some continuous positive definite functions $\alpha_{1}, \alpha_{2}, \alpha_{0} \in \mathcal{K}$. If we take the derivative of $V_{0}$ along the trajectories of the whole system (1), we have

$$
\begin{aligned}
& \frac{\partial V_{0}}{\partial t}+\frac{\partial V_{0}}{\partial x} f(t, x, u)=\frac{\partial V_{0}}{\partial t}+\frac{\partial V_{0}}{\partial x} f(t, x, 0) \\
& +\frac{\partial V_{0}}{\partial x}[f(t, x, u)-f(t, x, 0)]
\end{aligned}
$$

Since $V_{0}$ is continuously differentiable and we consider $x$ in a compact subset $D$, there exists a positive constant $K_{V_{0}}$ such that

$$
\left\|\frac{\partial V_{0}}{\partial x}\right\| \leq K_{V_{0}}, \quad \forall x \in \mathcal{D}
$$

Moreover, system (1) is locally Lipschitz in $x$ and $u$, uniformly in $t$. This implies that there exists a positive constant $L(x, u)$ such that

$$
\|f(t, x, u)-f(t, x, 0)\| \leq L(x, u)\|u\|
$$

$\forall x \in \mathcal{D}, \forall u \in \mathcal{D}_{u}, \forall t \geq 0$. Since $x \in \mathcal{D}, u \in \mathcal{D}_{u}$, where $\mathcal{D}, \mathcal{D}_{u}$ are compact, $L_{u_{\max }}:=$ 
$\max _{x \in \mathcal{D}, u \in \mathcal{D}_{u}} L(x, u)$ exists. Thus, using the inequality (13), and the definitions for $K_{V_{0}}$ and $L_{u_{\max }}$, we have

$$
\begin{aligned}
& \frac{\partial V_{0}}{\partial t}+\frac{\partial V_{0}}{\partial x} f(t, x, 0)+\frac{\partial V_{0}}{\partial x}[f(t, x, u)-f(t, x, 0)] \\
& \leq-\alpha_{0}(\|x\|)+K_{V_{0}} L_{u_{\max }}\|u\|
\end{aligned}
$$

After defining the $\mathcal{K}$-function $\sigma_{0}(s)=K_{V_{0}} L_{u_{\max }} s$, for $s \in \mathbb{R}_{\geq 0}$, we rewrite (17) as

$$
\dot{V}_{0} \leq-\alpha_{0}(\|x\|)+\sigma_{0}(\|u\|)
$$

Thus, by Definition 2.2, $V_{0}$ is an iISS Lyapunov function for the system (1).

$\underline{2 a \Longrightarrow 1 a}$ : Consider the iISS Lyapunov function $V_{0}$ for system (1) satisfying (12) and (18). Then, by similar sufficiency discussion as in (Angeli (2000), p. 1088), system (1) is locally iISS.

Consider now the dynamical system (9), and let us define the state vector $\mathbf{z}:=\left[\begin{array}{lll}z_{1} & z_{2} & z_{3}\end{array}\right]^{T}=$ $\left[\begin{array}{lll}x & \dot{x} & i\end{array}\right]^{T}$. The objective of the control is to make the variables $\left(z_{1}, z_{2}\right)$ robustly track sufficiently smooth (at least $C^{2}$ ) time-varying position and velocity trajectories $z_{1}^{\text {ref }}(t), z_{2}^{\text {ref }}(t)=\frac{d z_{1}^{\text {ref }}(t)}{d t}$ that satisfy the following constraints: $z_{1}^{\text {ref }}\left(t_{0}\right)=z_{1_{\text {int }}}, z_{1}^{\text {ref }}\left(t_{f}\right)=z_{1_{f}}, \dot{z}_{1}^{\text {ref }}\left(t_{0}\right)=\dot{z}_{1}^{\text {ref }}\left(t_{f}\right)=$ $0, \ddot{z}_{1}^{r e f}\left(t_{0}\right)=\ddot{z}_{1}^{r e f}\left(t_{f}\right)=0$, where $t_{0}$ is the starting time of the trajectory, $t_{f}$ is the final time, $z_{1_{\text {int }}}$ is the initial position and $z_{1_{f}}$ is the final position.

To start, let us first write the system (9) in the following form:

$$
\begin{aligned}
& \dot{z}_{1}=z_{2} \\
& \dot{z}_{2}=\frac{k}{m}\left(x_{0}-z_{1}\right)-\frac{\eta}{m} z_{2}-\frac{a}{2 m\left(b+z_{1}\right)^{2}} z_{3}^{2}+\frac{f_{d}}{m} \\
& \dot{z}_{3}=-\frac{R}{\frac{a}{b+z_{1}}} z_{3}+\frac{z_{3}}{b+z_{1}} z_{2}+\frac{u}{\frac{a}{b+z_{1}}}
\end{aligned}
$$

We consider the case where (19) has uncertainties on the spring constant $k$, the damping coefficient $\eta$, and the additive disturbance $f_{d}$. To take into account these uncertain coefficients, 
a backstepping controller is designed as (refer to the constructive proof of Lemma 2):

$$
\begin{aligned}
& u=\frac{a}{b+z_{1}}\left(\frac{R\left(b+z_{1}\right)}{a} z_{3}-\frac{z_{2} z_{3}}{\left(b+z_{1}\right)}+\frac{1}{2 z_{3}}\left(\frac{a}{2 m\left(b+z_{1}\right)^{2}}\left(z_{2}-z_{2}^{r e f}\right)-c_{2}\left(z_{3}^{2}-\tilde{u}\right)\right)\right) \\
& +\frac{2 m z_{2}}{z_{3}}\left(\frac{\hat{k}}{m}\left(x_{0}-z_{1}\right)-\frac{\hat{\eta}}{m} z_{2}+\frac{\hat{f}_{d}}{m}+c_{3}\left(z_{1}-z_{1}^{r e f}\right)+c_{1}\left(z_{2}-z_{2}^{r e f}\right)+\kappa_{1}\left(z_{2}-z_{2}^{r e f}\right)\|\psi\|_{2}^{2}-\dot{z}_{2}^{r e f}\right) \\
& +\frac{m\left(b+z_{1}\right)}{z_{3}}\left(\left(\frac{\hat{k}}{m}\left(x_{0}-z_{1}\right)-\frac{\hat{\eta}}{m} z_{2}+\frac{\hat{f}_{d}}{m}-\frac{a}{2 m\left(b+z_{1}\right)^{2}} z_{3}^{2}-\dot{z}_{2}^{r e f}\right)\left(c_{1}+\kappa_{1}\|\psi\|_{2}^{2}-\frac{\hat{\eta}}{m}\right)-\frac{\hat{\eta}}{m} \dot{z}_{2}^{r e f}\right) \\
& +\frac{m\left(b+z_{1}\right)}{z_{3}}\left(2 \kappa_{1}\left(z_{2}-z_{2}^{r e f}\right)\left(\frac{\left(x_{0}-z_{1}\right)\left(-z_{2}\right)}{m^{2}}+\frac{z_{2}\left(\frac{\hat{k}}{m}\left(x_{0}-z_{1}\right)-\frac{\hat{\eta}}{m} z_{2}+\frac{\hat{f}_{d}}{m}-\frac{a z_{3}^{2}}{m^{2}}\right)}{2 m\left(b+z_{1}\right)^{2}}\right)\right) \\
& -\kappa_{2}\left(z_{3}^{2}-\tilde{u}\right)\left|\frac{2 m\left(b+z_{1}\right)^{2}}{a}\right|^{2}\left[\left|c_{1}+\kappa_{1}\|\psi\|_{2}^{2}-\frac{\hat{\eta}}{m}\right|^{2}+\left|2 \kappa_{1}\left(z_{2}-z_{2}^{r e f}\right)\right|^{2}\left|\frac{z_{2}}{m^{2}}\right|^{2}\right]\|\psi\|_{2}^{2} \\
& -\kappa_{3}\left(z_{3}^{2}-\tilde{u}\right)\left|\frac{2 m\left(b+z_{1}\right)^{2}}{a}\right|^{2}\|\psi\|_{2}^{2}+\frac{m\left(b+z_{1}\right)}{z_{3}}\left(-\frac{\hat{k}}{m} z_{2}-\ddot{z}_{2}^{r e f}+c_{3}\left(z_{2}-z_{2}^{r e f}\right)\right)
\end{aligned}
$$

with

$$
\begin{aligned}
& \tilde{u}=\frac{2 m\left(b+z_{1}\right)^{2}}{a}\left(\frac{\hat{k}}{m}\left(x_{0}-z_{1}\right)-\frac{\hat{\eta}}{m} z_{2}+\frac{\hat{f}_{d}}{m}+c_{3}\left(z_{1}-z_{1}^{r e f}\right)+c_{1}\left(z_{2}-z_{2}^{r e f}\right)-\dot{z}_{2}^{r e f}\right) \\
& +\frac{2 m\left(b+z_{1}\right)^{2}}{a}\left(\kappa_{1}\left(z_{2}-z_{2}^{r e f}\right)\|\psi\|_{2}^{2}\right)
\end{aligned}
$$

where $\hat{k}, \hat{\eta}, \hat{f}_{d}$ are the system parameter estimates, and $\psi \triangleq\left[\frac{x_{0}-z_{1}}{m} \frac{z_{2}}{m} \frac{1}{m}\right]^{T}$. We can now state the following lemma.

\section{Lemma 2}

Consider the closed-loop dynamics given by (19), (20) and (21), with constant unknown parameters $k, \eta, f_{d}$ and consider the parameter estimation error vector $\Delta \triangleq\left[k-\hat{k} \eta-\hat{\eta} f_{d}-\hat{f}_{d}\right]^{T}$. Then, there exist positive gains $c_{1}, c_{2}, c_{3}, \kappa_{1}, \kappa_{2}$ and $\kappa_{3}$ such that $\left(z_{1}(t), z_{2}(t)\right)$ are uniformly bounded and the system (19) is locally integral input-to state stable (LiISS) with respect to $(\Delta, \dot{\Delta})$.

Proof: Consider the mechanical subsystem that consists of the first two equations, where we define the virtual control input $\tilde{u}:=z_{3}^{2}$

$$
\begin{aligned}
& \dot{z}_{1}=z_{2} \\
& \dot{z}_{2}=\frac{k}{m}\left(x_{0}-z_{1}\right)-\frac{\eta}{m} z_{2}+\frac{f_{d}}{m}-\frac{a}{2 m\left(b+z_{1}\right)^{2}} \tilde{u}
\end{aligned}
$$

We define the Lyapunov function $V_{\text {sub }}=\frac{c_{3}}{2}\left(z_{1}-z_{1}^{\text {ref }}\right)^{2}+\frac{1}{2}\left(z_{2}-z_{2}^{\text {ref }}\right)^{2}$, with $c_{3}>0$. To ensure that $\dot{V}_{\text {sub }}$ is upper bounded by a quadratic function of the tracking error and the uncertain 
parameters estimation error, we design $\tilde{u}$ as given in equation (21), which leads to

$$
\begin{aligned}
& \dot{V}_{\text {sub }}=-c_{3}\left(z_{1}-z_{1}^{\text {ref }}\right)\left(\dot{z}_{1}-\dot{z}_{1}^{r e f}\right)+\left(z_{2}-z_{2}^{r e f}\right)\left(\dot{z}_{2}-\dot{z}_{2}^{r e f}\right) \\
& =\left(z_{2}-z_{2}^{r e f}\right)\left(c_{3}\left(z_{1}-z_{1}^{r e f}\right)+\frac{k}{m}\left(x_{0}-z_{1}\right)-\frac{\eta}{m} z_{2}+\frac{f_{d}}{m}-\dot{z}_{2}^{r e f}-\frac{a}{2 m\left(b+z_{1}\right)^{2}} \tilde{u}\right) \\
& =-c_{1}\left(z_{2}-z_{2}^{r e f}\right)^{2}+\left(z_{2}-z_{2}^{r e f}\right)\left(\frac{(k-\hat{k})\left(x_{0}-z_{1}\right)}{m}-\frac{(\eta-\hat{\eta}) z_{2}}{m}+\frac{f_{d}-\hat{f}_{d}}{m}\right)-\kappa_{1}\left(z_{2}-z_{2}^{r e f}\right)^{2}\|\psi\|_{2}^{2}
\end{aligned}
$$

Using the definitions of the vectors $\psi$ and $\Delta$, we have

$$
\begin{aligned}
& \dot{V}_{\text {sub }} \leq c_{1}\left(z_{2}-z_{2}^{r e f}\right)^{2}+\left|z_{2}-z_{2}^{r e f}\right|\left\|\psi^{T}\right\|_{2}\|\Delta\|_{2}-\kappa_{1}\left(z_{2}-z_{2}^{r e f}\right)^{2}\|\psi\|_{2}^{2} \\
& \leq-c_{1}\left(z_{2}-z_{2}^{r e f}\right)^{2}-\kappa_{1}\left[\left|z_{2}-z_{2}^{r e f}\right|\|\psi\|_{2}-\frac{\|\Delta\|_{2}}{2 \kappa_{1}}\right]^{2}+\frac{\|\Delta\|_{2}^{2}}{4 \kappa_{1}} \\
& \leq-c_{1}\left(z_{2}-z_{2}^{r e f}\right)^{2}+\frac{\|\Delta\|_{2}^{2}}{4 \kappa_{1}}
\end{aligned}
$$

where $\Delta=\left[k-\hat{k} \eta-\hat{\eta} f_{d}-\hat{f}_{d}\right]^{T}$ is the vector holding the discrepancy between the actual system parameters and the estimated parameters. Note that we have made use of the nonlinear damping term $-\kappa_{1}\left(z_{2}-z_{2}^{r e f}\right)^{2}\|\psi\|_{2}^{2}$ to attain a negative quadratic term of $\psi$ and $\Delta$ $\left(\right.$ i.e., $\left.-\kappa_{1}\left[\left|z_{2}-z_{2}^{r e f}\right|\|\psi\|_{2}-\frac{\|\Delta\|_{2}}{2 \kappa_{1}}\right]^{2}\right)$ and a positive term that is function of $\Delta$ only, i.e., $\frac{\|\Delta\|_{2}^{2}}{4 \kappa_{1}}$ (refer to Krstic (1995) for a detailed presentation of the use of nonlinear damping terms in Lyapunov analysis to achieve ISS stability). Since we cannot directly control $z_{3}^{2}$, we use backstepping to design the control input $u(t)$ so that $z_{3}^{2}$ converges to $\tilde{u}$, which in turn will render the mechanical subsystem LiISS, as proven below. To this purpose, we define the Lyapunov function for the full system: $V_{\text {aug }}=V_{\text {sub }}+\frac{\left(z_{3}^{2}-\tilde{u}\right)^{2}}{2}$. Taking the derivative of $V_{\text {aug }}$ along the trajectories of the full system, leads to the following inequality

$$
\begin{aligned}
& \dot{V}_{\text {aug }} \leq-c_{1}\left(z_{2}-z_{2}^{r e f}\right)^{2}+\frac{\|\Delta\|_{2}^{2}}{4 \kappa_{1}}+\left(z_{3}^{2}-\tilde{u}\right)\left(-\frac{a\left(z_{2}-z_{2}^{r e f}\right)}{2 m\left(b+z_{1}\right)^{2}}-\dot{\tilde{u}}\right) \\
& +\left(z_{3}^{2}-\tilde{u}\right)\left(2 z_{3}\left(-\frac{R\left(b+z_{1}\right)}{a} z_{3}+\frac{z_{2} z_{3}}{\left(b+z_{1}\right)}+\frac{b+z_{1}}{a} u\right)\right)
\end{aligned}
$$

where $\dot{\tilde{u}}$ is obtained from $(21)$ as

$$
\begin{aligned}
& \dot{\tilde{u}}=\frac{4 m\left(b+z_{1}\right) z_{2}}{a}\left(\frac{\hat{k}}{m}\left(x_{0}-z_{1}\right)-\frac{\hat{\eta}}{m} z_{2}+\frac{\hat{f}_{d}}{m}+c_{3}\left(z_{1}-z_{1}^{r e f}\right)+c_{1}\left(z_{2}-z_{2}^{r e f}\right)\right) \\
& +\frac{4 m\left(b+z_{1}\right) z_{2}}{a}\left(\kappa_{1}\left(z_{2}-z_{2}^{r e f}\right)\|\psi\|_{2}^{2}-\dot{z}_{2}^{r e f}\right) \\
& +\frac{2 m\left(b+z_{1}\right)^{2}}{a}\left(\frac{\dot{\hat{k}}}{m}\left(x_{0}-z_{1}\right)-\frac{\dot{\hat{\eta}}}{m} z_{2}+\frac{\dot{\hat{f}}_{d}}{m}\right) \\
& +\frac{2 m\left(b+z_{1}\right)^{2}}{a}\left(\left(\frac{k}{m}\left(x_{0}-z_{1}\right)-\frac{\eta}{m} z_{2}+\frac{f_{d}}{m}-\frac{a}{2 m\left(b+z_{1}\right)^{2}} z_{3}^{2}-\dot{z}_{2}^{r e f}\right)\left(c_{1}+\kappa_{1}\|\psi\|_{2}^{2}-\frac{\hat{\eta}}{m}\right)-\frac{\hat{\eta}}{m} \dot{z}_{2}^{r e f}\right) \\
& +\frac{2 m\left(b+z_{1}\right)^{2}}{a}\left(2 \kappa_{1}\left(z_{2}-z_{2}^{r e f}\right)\left(\frac{\left(x_{0}-z_{1}\right)\left(-z_{2}\right)}{m^{2}}+\frac{z_{2}\left(\frac{k}{m}\left(x_{0}-z_{1}\right)-\frac{\eta}{m} z_{2}+\frac{f_{d}}{m}-\frac{a z_{3}^{2}}{m^{2}}\right)}{2 m\left(b+z_{1}\right)^{2}}\right)\right) \\
& +\frac{2 m\left(b+z_{1}\right)^{2}}{a}\left(-\frac{\hat{k}}{m} z_{2}-\ddot{z}_{2}^{r e f}+c_{3}\left(z_{2}-z_{2}^{r e f}\right)\right)
\end{aligned}
$$

The goal now is to write an upper bound of $\dot{V}_{\text {aug }}$ as a sum of quadratic terms of the tracking errors, the error $z_{3}^{2}-\tilde{u}$, and the uncertain parameters estimation error. By choosing the control 
input as (20), equation (25) becomes

$$
\begin{aligned}
& \dot{V}_{\text {aug }} \leq-c_{1}\left(z_{2}-z_{2}^{r e f}\right)^{2}+\frac{\|\Delta\|_{2}^{2}}{4 \kappa_{1}}-c_{2}\left(z_{3}^{2}-\tilde{u}\right)^{2} \\
& -\left(z_{3}^{2}-\tilde{u}\right)\left(\frac{2 m\left(b+z_{1}\right)^{2}}{a}\left(\frac{(k-\hat{k})\left(x_{0}-z_{1}\right)}{m}-\frac{(\eta-\hat{\eta}) z_{2}}{m}+\frac{f_{d}-\hat{f}_{d}}{m}\right)\left(c_{1}+\kappa_{1}\|\psi\|_{2}^{2}-\frac{\hat{\eta}}{m}\right)\right) \\
& -\left(z_{3}^{2}-\tilde{u}\right)\left(2 \kappa_{1}\left(z_{2}-z_{2}^{r e f}\right)\left(\frac{2 z_{2}\left(b+z_{1}\right)^{2}}{m a}\right)\left(\frac{(k-\hat{k})\left(x_{0}-z_{1}\right)}{m}-\frac{(\eta-\hat{\eta}) z_{2}}{m}+\frac{f_{d}-\hat{f}_{d}}{m}\right)\right) \\
& -\left(z_{3}^{2}-\tilde{u}\right)\left(\frac{2 m\left(b+z_{1}\right)^{2}}{a}\right)\left(\frac{\hat{\hat{k}}}{m}\left(x_{0}-z_{1}\right)-\frac{\dot{\hat{\eta}}}{m} z_{2}+\frac{\dot{\hat{f}}}{m}\right) \\
& -\kappa_{3}\left(z_{3}^{2}-\tilde{u}\right)^{2}\left|\frac{2 m\left(b+z_{1}\right)^{2}}{a}\right|^{2}\|\psi\|_{2}^{2} \\
& -\kappa_{2}\left(z_{3}^{2}-\tilde{u}\right)^{2}\left[\left|\frac{2 m\left(b+z_{1}\right)^{2}}{a}\right|^{2}\left|c_{1}+\kappa_{1}\|\psi\|_{2}^{2}-\frac{\hat{\eta}}{m}\right|^{2}+\left|2 \kappa_{1}\left(z_{2}-z_{2}^{r e f}\right)\right|^{2}\left|\frac{2 z_{2}\left(b+z_{1}\right)^{2}}{m a}\right|^{2}\right]\|\psi\|_{2}^{2}
\end{aligned}
$$

Using the aforementioned definitions of the vectors $\psi$ and $\Delta$, and noting that $\dot{\Delta}=$ $\left[-\dot{\hat{k}}-\dot{\hat{\eta}}-\dot{\hat{f}}_{d}\right]^{T}$, we can further bound $\dot{V}_{a u g}$ in the following way

$$
\begin{aligned}
& \dot{V}_{\text {aug }} \leq-c_{1}\left(z_{2}-z_{2}^{r e f}\right)^{2}+\frac{\|\Delta\|_{2}^{2}}{4 \kappa_{1}}-c_{2}\left(z_{3}^{2}-\tilde{u}\right)^{2} \\
& +\left|z_{3}^{2}-\tilde{u}\left\|\frac{2 m\left(b+z_{1}\right)^{2}}{a}|| c_{1}+\kappa_{1}\right\| \psi\left\|_{2}^{2}-\frac{\hat{\eta}}{m} \mid\right\| \psi^{T}\left\|_{2}\right\| \Delta \|_{2}\right. \\
& +\left|z_{3}^{2}-\tilde{u}\left\|2 \kappa_{1}\left(z_{2}-z_{2}^{r e f}\right)|| \frac{2 z_{2}\left(b+z_{1}\right)^{2}}{m a} \mid\right\| \psi^{T}\left\|_{2}\right\| \Delta \|_{2}\right. \\
& +\left|z_{3}^{2}-\tilde{u}\left\|\frac{2 m\left(b+z_{1}\right)^{2}}{a} \mid\right\| \psi^{T}\left\|_{2}\right\| \dot{\Delta} \|_{2}\right. \\
& -\kappa_{3}\left(z_{3}^{2}-\tilde{u}\right)^{2}\left|\frac{2 m\left(b+z_{1}\right)^{2}}{a}\right|^{2}\|\psi\|_{2}^{2} \\
& -\kappa_{2}\left(z_{3}^{2}-\tilde{u}\right)^{2}\left[\left|\frac{2 m\left(b+z_{1}\right)^{2}}{a}\right|^{2}\left|c_{1}+\kappa_{1}\|\psi\|_{2}^{2}-\frac{\hat{\eta}}{m}\right|^{2}+\left|2 \kappa_{1}\left(z_{2}-z_{2}^{r e f}\right)\right|^{2}\left|\frac{2 z_{2}\left(b+z_{1}\right)^{2}}{m a}\right|^{2}\right]\|\psi\|_{2}^{2}
\end{aligned}
$$

By making use of the quadratic damping terms, e.g. $-\kappa_{1}\left(z_{2}-z_{2}^{r e f}\right)^{2}\|\psi\|_{2}^{2}$, we can further simplify the right hand-side of the previous inequality. For instance, if we consider the term

$$
\left|z_{3}^{2}-\tilde{u}\right|\left|2 \kappa_{1}\left(z_{2}-z_{2}^{r e f}\right)\right|\left|\frac{2 z_{2}\left(b+z_{1}\right)^{2}}{m a}\right|\left\|\psi^{T}\right\|_{2}\|\Delta\|_{2}-\kappa_{2}\left(z_{3}^{2}-\tilde{u}\right)^{2}\left|2 \kappa_{1}\left(z_{2}-z_{2}^{r e f}\right)\right|^{2}\left|\frac{2 z_{2}\left(b+z_{1}\right)^{2}}{m a}\right|^{2}\|\psi\|_{2}^{2}
$$

we can write is as

$$
\begin{aligned}
& \left|z_{3}^{2}-\tilde{u}\right|\left|2 \kappa_{1}\left(z_{2}-z_{2}^{r e f}\right)\right|\left|\frac{2 z_{2}\left(b+z_{1}\right)^{2}}{m a}\right|\left\|\psi^{T}\right\|_{2}\|\Delta\|_{2}-\kappa_{2}\left(z_{3}^{2}-\tilde{u}\right)^{2}\left|2 \kappa_{1}\left(z_{2}-z_{2}^{r e f}\right)\right|^{2}\left|\frac{2 z_{2}\left(b+z_{1}\right)^{2}}{m a}\right|^{2}\|\psi\|_{2}^{2} \\
& +\frac{\|\Delta\|_{2}^{2}}{4 \kappa_{2}}-\frac{\|\Delta\|_{2}^{2}}{4 \kappa_{2}}
\end{aligned}
$$

which finally equals to

$$
-\kappa_{2}\left[\left|z_{3}^{2}-\tilde{u}\right|\left|2 \kappa_{1}\left(z_{2}-z_{2}^{r e f}\right)\right|\left|\frac{2 z_{2}\left(b+z_{1}\right)^{2}}{m a}\right|\|\psi\|_{2}-\frac{\|\Delta\|_{2}}{2 \kappa_{2}}\right]^{2}+\frac{\|\Delta\|_{2}^{2}}{4 \kappa_{2}}
$$


following the same steps for the remaining terms in (28), we get

$$
\begin{aligned}
& \dot{V}_{\text {aug }} \leq-c_{1}\left(z_{2}-z_{2}^{r e f}\right)^{2}+\frac{\|\Delta\|_{2}^{2}}{4 \kappa_{1}}-c_{2}\left(z_{3}^{2}-\tilde{u}\right)^{2} \\
& -\kappa_{2}\left[\left|z_{3}^{2}-\tilde{u}\left\|\frac{2 m\left(b+z_{1}\right)^{2}}{a}|| c_{1}+\kappa_{1}\right\| \psi\left\|_{2}^{2}-\frac{\hat{\eta}}{m} \mid\right\| \psi \|_{2}-\frac{\|\Delta\|_{2}}{2 \kappa_{2}}\right]^{2}+\frac{\|\Delta\|_{2}^{2}}{4 \kappa_{2}}\right. \\
& -\kappa_{2}\left[\left|z_{3}^{2}-\tilde{u}\left\|2 \kappa_{1}\left(z_{2}-z_{2}^{r e f}\right)\right\| \frac{2 z_{2}\left(b+z_{1}\right)^{2}}{m a}\right|\|\psi\|_{2}-\frac{\|\Delta\|_{2}}{2 \kappa_{2}}\right]^{2}+\frac{\|\Delta\|_{2}^{2}}{4 \kappa_{2}} \\
& -\kappa_{3}\left[\left|z_{3}^{2}-\tilde{u}\left\|\frac{2 m\left(b+z_{1}\right)^{2}}{a} \mid\right\| \psi \|_{2}-\frac{\|\dot{\Delta}\|_{2}}{2 \kappa_{3}}\right]^{2}+\frac{\|\dot{\Delta}\|_{2}^{2}}{4 \kappa_{3}}\right.
\end{aligned}
$$

Finally, from (32), we deduce

$$
\dot{V}_{\text {aug }} \leq-c_{1}\left(z_{2}-z_{2}^{r e f}\right)^{2}-c_{2}\left(z_{3}^{2}-\tilde{u}\right)^{2}+\left(\frac{1}{4 \kappa_{1}}+\frac{1}{2 \kappa_{2}}\right)\|\Delta\|_{2}^{2}+\frac{\|\dot{\Delta}\|_{2}^{2}}{2 \kappa_{3}}
$$

It is easy to see that the uncertain system can be expressed in the following nonlinear timevarying form

$$
\dot{e}=f(t, e, \tilde{\Delta})
$$

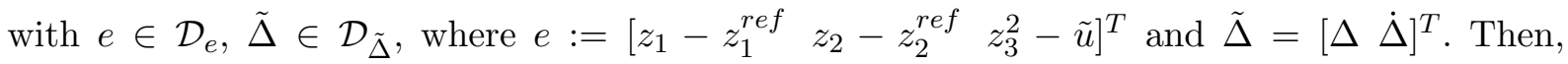
considering the output map defined by $h=\left[\begin{array}{ll}z_{2}-z_{2}^{\text {ref }} & z_{3}^{2}-\tilde{u}\end{array}\right]^{T}$, we show now that the system (34) with $h$ is weakly zero-detectable, by analyzing the zero-dynamics of (34) with $h \equiv \tilde{\Delta} \equiv 0$ ). Indeed, $\tilde{\Delta} \equiv 0$ means that we are analyzing the zero dynamics of the feedback system in the nominal case. Now considering the output condition $h \equiv 0$, together with the dynamics (21), and (22), leads to the following zero dynamics

$$
\begin{aligned}
& \dot{z}_{1}=z_{2} \\
& \dot{z}_{2}=\dot{z}_{2}^{\text {ref }}-c_{3}\left(z_{1}-z_{1}^{r e f}\right)-c_{1}\left(z_{2}-z_{2}^{r e f}\right)
\end{aligned}
$$

Writing the second equation in (35) in terms of $z_{1}$ and $z_{1}^{r e f}$ only, and introducing $e_{z_{1}}:=z_{1}-z_{1}^{r e f}$, we obtain

$$
\ddot{e}_{z_{1}}+c_{1} \dot{e}_{z_{1}}+c_{3} e_{z_{1}}=0
$$

It can be seen that if $c_{3}$ and $c_{1}$ are selected such that

$$
-c_{1} \pm \sqrt{c_{1}^{2}-4 c_{3}}<0
$$

the roots of the characteristic equation of (36) would be negative, which in turn would imply $\lim _{t \rightarrow \infty} z_{1}=z_{1}^{r e f}$ starting from any initial condition $z_{1}\left(t_{0}\right)$. Furthermore, inequality (33) satisfies (7), meaning that property $3 a$ holds for (34). By the virtue of Lemma 4.2, we conclude that system (34) is LiISS with respect to the input $\tilde{\Delta}$, implying that there exist functions $\alpha \in \mathcal{K}$, 
$\beta \in \mathcal{K} \mathcal{L}$ and $\gamma \in \mathcal{K}$, such that, for all $e(0) \in \mathcal{D}_{e}$ and $\tilde{\Delta} \in \mathcal{D}_{\tilde{\Delta}}$, and

$$
\|e(t)\| \leq \beta(\|e(0)\|, t)+\alpha\left(\int_{0}^{t} \gamma(\|\tilde{\Delta}\|)\right) d s
$$

for all $t \geq 0$.

In the rest of this paper, we will refer to the controller of Section 4.1 as the ISS-backstepping controller.

Remark 2: We underline here that, in this paper we assume constant unknown parameters $k, \eta, f_{d}$. This is a realistic assumption since we are targeting the problem of aging, which usually happens very slowly over long period of time. Hence, the slowly varying parameters can be approximated by constant uncertain parameters.

The analysis of the dynamical behavior of estimated parameters is done via multi-variable extremum seeking theory (Ariyur and Krstić (2003), Ariyur and Krstic (2002)). This analysis is described in the next section.

\subsection{Robustification of the ISS-backstepping Controller}

We will now discuss how multi-variable extremum seeking scheme is utilized along with ISSbackstepping controller to improve the controller robustness with respect to uncertainties in the system parameters. We define a performance cost function for the dynamical system (19) as

$$
Q(\theta)=q_{1}\left(z_{1}\left(t_{f}\right)-z_{1}\left(t_{f}\right)^{r e f}\right)^{2}+q_{2}\left(z_{2}\left(t_{f}\right)-z_{2}^{r e f}\left(t_{f}\right)\right)^{2}, q_{1}, q_{2}>0
$$

where, $\theta=\left(\theta_{1}, \theta_{2}, \theta_{3}\right)^{T}$ represents the vector of the learned parameters, defined such that

$$
\begin{aligned}
& \hat{k}(t)=k_{\text {nominal }}+\theta_{1}(t) \\
& \hat{\eta}(t)=\eta_{\text {nominal }}+\theta_{2}(t) \\
& \hat{f}_{d}(t)=f_{d-\text { nominal }}+\theta_{3}(t)
\end{aligned}
$$

with $k_{\text {nominal }}, \eta_{\text {nominal }}, f_{d-\text { nominal }}$ being the nominal values of the parameters.

To be able to derive some closed-form analysis of the learning algorithm, we need the following additional assumptions.

Assumption 4.3 The cost function $Q$ has a local minimum at $\theta^{*}=\left[k-k_{\text {nominal }} \eta-\eta_{\text {nominal }} f_{d}-\right.$ $\left.f_{d-\text { nominal }}\right]^{T}$. 
Assumption 4.4 The initial error $\Delta\left(t_{0}\right)$ is sufficiently small, i.e., the original parameters estimates vector $\left[\hat{k} \hat{\eta} \hat{f}_{d}\right]^{T}$ is close enough to the actual parameters vector $\left[k \eta f_{d}\right]^{T}$.

Assumption 4.5 The cost function is analytic and its variation with respect to the uncertain variables is bounded in the neighborhood of $\theta^{*}$, i.e., $\left\|\frac{\partial Q}{\partial \theta}(\tilde{\theta})\right\| \leq \xi_{2}, \xi_{2}>0, \tilde{\theta} \in \mathcal{V}\left(\theta^{*}\right)$, where $\mathcal{V}\left(\theta^{*}\right)$ denotes a compact neighborhood of $\theta^{*}$.

Remark 3: Assumption 4.3 simply means that we assume that $Q$ has at least a local minimum at the true values of the coefficients $k \eta, f_{d}$. Indeed, if we consider the iISS results proven in Lemma 2, equation (38) shows that if we assume the ideal case where we have $\hat{k}=k, \hat{\eta}=\eta, \hat{f}_{d}=f_{d}$, we have convergence of the actual trajectories to the desired trajectories, which means convergence of the cost function to zero. In other words, in the case of certain model, the ISS backstepping control reduces to a classical backstepping controller which achieves the desired trajectory tracking, i.e., minimum value for $Q$.

Remark 4: Assumption 4.4 indicates that our result will be of local nature, meaning that our analysis holds in a small neighborhood of the actual values of the parameters. Indeed, this assumption is realistic for the targeted application, i.e., estimation of uncertainties due to aging, since in the context of aging the uncertain parameters vary slowly over a long period of time, and the controller starts initially from a good guess of the parameters given by their nominal values (before aging), and subsequently follows the drift of the parameters over time.

Remark 5: The choice of the cost function $Q$ is not unique. Indeed, we choose the form (39), because we are mainly interested in minimizing the position and velocity errors at $t=t_{f}$ to improve the soft landing performance, however, if we were interested in improving the tracking performance over the whole interval $\left[0, t_{f}\right]$, we could choose the cost function $Q(\theta)=\int_{0}^{t_{f}} q_{1}\left(z_{1}(s)-z_{1}(s)^{r e f}\right)^{2} d s+\int_{0}^{t_{f}} q_{2}\left(z_{2}(s)-z_{2}^{r e f}(s)\right)^{2} d s, q_{1}, q_{2}>0$.

Following Rotea (2000), Ariyur and Krstić (2003), we propose the following MES algorithm for the system (19):

$$
\begin{aligned}
& \dot{x}_{p}=a_{p} \sin \left(\omega_{p} t+\frac{\pi}{2}\right) Q\left(\theta_{p}\right) \\
& \theta_{p}=x_{p}+a_{p} \sin \left(\omega_{p} t-\frac{\pi}{2}\right), p=1,2,3
\end{aligned}
$$

where $p=1$ corresponds to $k, p=2$ corresponds to $\eta$ and $p=3$ corresponds to $f_{d}$, $a_{p}>0, p=1,2,3$ and $\omega_{i} \neq \omega_{j}, \omega_{i}+\omega_{j} \neq \omega_{k}, i, j, k \in\{1,2,3\}, \omega_{i}>\omega^{*}, \forall i \in\{1,2,3\}$, with $\omega^{*}$ large enough. The algorithm (41) is actually a special case of the algorithms proposed in Rotea (2000), Ariyur and Krstić (2003), where we replace by a unit transfer function the filters 
used in the general algorithms presented in Rotea (2000), Ariyur and Krstić (2003). Indeed, these filters are mainly introduced in Rotea (2000), Ariyur and Krstić (2003) to accelerate the convergence of the MES algorithm. For simplicity of the presentation, we choose to use a version of the algorithms without the filters. This version can be seen as an extension to the multi-variable case of the single-variable extremum seeking algorithm used in Krstic and Wang (2000), Peterson and Stefanopoulou (2004). The uncertain parameters estimates are then updated following (40). Here due to the cyclic nature of the problem, the uncertain parameters estimate vector $\left(\hat{k}, \hat{\eta}, \hat{f}_{d}\right)^{T}$ is updated for each cycle, i.e., at the end of each cycle at $t=t_{f}$, the cost function $Q$ is updated. Then, the new estimate of the parameters is computed for the next cycle, starting from the same states' initial condition. The purpose of using MES scheme along with ISS-backstepping controller is to improve the performance of the ISS-backstepping controller by better estimating the system parameters over many cycles, hence decreasing the error in the parameters over time to provide better trajectory following for the actuator.

Now we can state the main result about the MES-based adaptive controller.

\section{Lemma 3}

Consider the dynamical system (19) with the ISS-backstepping controller given by (20) and (21). Moreover, consider the cycle-to-cycle multi-variable extremum seeking algorithm for estimation of system parameters $k, \eta$, and $f_{d}$, described by (39), (40) and (41). Then, under

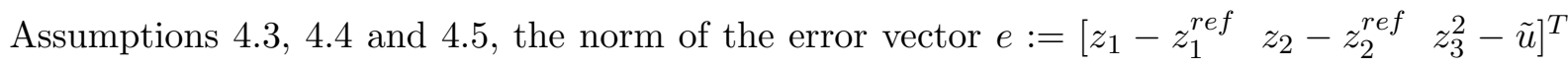
admits the following bound

$$
\|e(t)\| \leq \beta(\|e(0)\|, t)+\alpha\left(\int_{0}^{t} \gamma\left(\tilde{\beta}(\|\Delta(0)\|, t)+\|\tilde{\Delta}\|_{\max }\right)\right) d s
$$

where, $\|\tilde{\Delta}\|_{\max }=\frac{\xi_{1}}{\omega_{0}}+\sqrt{\sum_{i=1}^{i=3} a_{i}^{2}}\left(1+\omega_{0}\right)+\max _{i \in\{1,2,3\}} 0.5 \xi_{2} a_{i}^{2}, \xi_{1}, \xi_{2}>0, e(0) \in \mathcal{D}_{e}, \omega_{0}=$ $\max _{i \in\{1,2,3\}} \omega_{i}, \alpha \in \mathcal{K}, \beta \in \mathcal{K} \mathcal{L}, \tilde{\beta} \in \mathcal{K} \mathcal{L}$ and $\gamma \in \mathcal{K}$.

Proof:

The first part of the proof relies on the result of Lemma 2. Indeed, based on Lemma 2, we know that for the closed-loop dynamics given by (19), (20) and (21), there exist functions $\alpha \in \mathcal{K}$, $\beta \in \mathcal{K} \mathcal{L}$ and $\gamma \in \mathcal{K}$, such that, for all $e(0) \in \mathcal{D}_{e}$ and $\tilde{\Delta} \in \mathcal{D}_{\tilde{\Delta}}$, the norm of the error vector

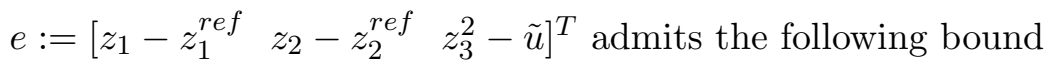

$$
\|e(t)\| \leq \beta(\|e(0)\|, t)+\alpha\left(\int_{0}^{t} \gamma(\|\tilde{\Delta}\|)\right) d s
$$

for all $t \geq 0$.

Now, we need to evaluate the bound on the estimation vector $\tilde{\Delta}$; to do so we use the results 
presented in Rotea (2000). First, based on Assumption 4.5, the cost function is locally Lipschitz, i.e., $\exists \eta_{1}>0$, s.t. $\left|Q\left(\theta_{1}\right)-Q\left(\theta_{2}\right)\right| \leq \eta_{1}\left\|\theta_{1}-\theta_{2}\right\|, \forall \theta_{1}, \theta_{2} \in \mathcal{V}\left(\theta^{*}\right)$. Furthermore, since $Q$ is analytic it can be approximated locally in $\mathcal{V}\left(\theta^{*}\right)$ with a quadratic function, e.g. Taylor series up to second order. Based on this and on Assumptions 4.3 and 4.4, we can write the following bound (Rotea (2000), pages 436-437):

$$
\begin{gathered}
\|\Delta(t)\|-\|d(t)\| \leq\|\Delta(t)-d(t)\| \leq \tilde{\beta}(\|\Delta(0)\|, t)+\frac{\xi_{1}}{\omega_{0}} \\
\Rightarrow\|\Delta(t)\| \leq \tilde{\beta}(\|\Delta(0)\|, t)+\frac{\xi_{1}}{\omega_{0}}+\|d(t)\| \\
\Rightarrow\|\Delta(t)\| \leq \tilde{\beta}(\|\Delta(0)\|, t)+\frac{\xi_{1}}{\omega_{0}}+\sqrt{\sum_{i=1}^{i=3} a_{i}^{2}}
\end{gathered}
$$

with $\tilde{\beta} \in \mathcal{K} \mathcal{L}, \quad \xi_{1}>0, \quad t \geq 0, \omega_{0}=\max _{i \in\{1,2,3\}} \omega_{i}, d(t)=\left[a_{1} \sin \left(\omega_{1} t+\frac{\pi}{2}\right), a_{2} \sin \left(\omega_{2} t+\right.\right.$ $\left.\left.\frac{\pi}{2}\right), a_{3} \sin \left(\omega_{3} t+\frac{\pi}{2}\right)\right]^{T}$. Moreover, in (Rotea (2000), pages 434), the MES algorithm is shown to be a gradient-based algorithm, such that the variation of $\theta$ over time is approximated by

$$
\dot{\theta} \simeq-R \frac{\partial Q}{\partial \theta}(\theta)+\dot{d}(t)
$$

with $R=\lim _{T \rightarrow \infty} \int_{0}^{T}\|d(s)\|^{2} d s=0.5 \operatorname{diag}\left\{a_{1}^{2}, a_{2}^{2}, a_{3}^{2}\right\}$.

Using Assumption 4.5, we can write

$$
\|\dot{\theta}\|=\|\dot{\Delta}\| \leq 0.5 \max _{i \in\{1,2,3\}} a_{i}^{2} \xi_{2}+\sqrt{\sum_{i=1}^{i=3}\left(a_{i} \omega_{i}\right)^{2}} \leq 0.5 \max _{i \in\{1,2,3\}} a_{i}^{2} \xi_{2}+\sqrt{\sum_{i=1}^{i=3}\left(a_{i}\right)^{2} \omega_{0}}
$$

Finally, we can write the following bound on $\|\tilde{\Delta}\|$ :

$$
\begin{gathered}
\|\tilde{\Delta}\| \leq\|\Delta\|+\|\dot{\Delta}\| \\
\Rightarrow\|\tilde{\Delta}\| \leq \tilde{\beta}(\|\Delta(0)\|, t)+\frac{\xi_{1}}{\omega_{0}}+\sqrt{\sum_{i=1}^{i=3} a_{i}^{2}}\left(1+\omega_{0}\right)+\max _{i \in\{1,2,3\}} 0.5 \xi_{2} a_{i}^{2}
\end{gathered}
$$

which together with the bound (43) completes the proof.

Remark 6: The estimated parameters upper bounds used in Lemma 3 are correlated to the choice of the first order multi-variable extremum seeking (39), (40) and (41). However, these bounds can be easily changed by using other MES algorithms, e.g. Noase (2011), Scheinker (2013), which is due to the modular design of the controller, that uses the iISS robust part to ensure boundedness of the error dynamics and the learning part to improve the tracking performance.

Remark 7: To simplify the presentation, we choose here to use a first order extremumseeking algorithm, which has been shown in Rotea (2000) to be a gradient-based algorithm. 


\begin{tabular}{|c|c|}
\hline Parameter & Value \\
\hline$m$ & $0.27[\mathrm{~kg}]$ \\
\hline$R$ & $6[\Omega]$ \\
\hline$\eta$ & $7.53\left[\frac{\mathrm{kg}}{\mathrm{s}}\right]$ \\
\hline$x_{0}$ & $8[\mathrm{~mm}]$ \\
\hline$k$ & $158\left[\frac{\mathrm{N}}{\mathrm{mm}}\right]$ \\
\hline$a$ & $14.96 \times 16^{-6}\left[\frac{\mathrm{Nm^{2 }}}{A^{2}}\right]$ \\
\hline$b$ & $4 \times 10^{-5}[\mathrm{~m}]$ \\
\hline
\end{tabular}

Table 1.: System Parameter Values

Note that this is not to be confused with optimization methods that need an explicit computation or a numerical estimation of the gradient via numerical differentiation. Here, by gradient-based we mean that the step of the optimized variables are shown to be taken in the direction of the cost function's gradient without the need of an explicit computation of the gradient. However, due to the modular design of the proposed approach, one could use other non-gradient based extremum seekers, e.g. Nesic (2013), which achieve global extremum seeking on compact sets in the presence of local extrema.

\section{Simulations}

In this section, we illustrate our approach on a nonlinear electromagnetic actuator modelled by (9), using the system parameters given in Table 1 (Kahveci and Kolmanovsky (2010)). The reference trajectory is designed to be a $5^{\text {th }}$ order polynomial, $x^{r e f}(t)=\sum_{i=0}^{5} a_{i}\left(\frac{t}{t_{f}}\right)^{i}$ where the coefficients $a_{i}$ are selected such that the following conditions are satisfied: $x^{r e f}(0)=$ $0.2 \mathrm{~mm}, x^{r e f}(0.5)=0.7 \mathrm{~mm}, \dot{x}^{r e f}(0)=0, \dot{x}^{r e f}(0.5)=0, \ddot{x}^{r e f}(0)=0, \dot{x}^{r e f}(0.5)=0$. We present hereafter two cases to illustrate the performance of the proposed controller.

We consider the following uncertainty in the mechanical parameters $k, \eta$ and $f_{d}: \Delta k=-4.5$, $\Delta \eta=-0.7$ and $\Delta f_{d}=-7.5$. To make the simulation case more challenging, we also introduced an initial error $x(0)=0.01 \mathrm{~mm}$ on the armature position. We implemented the controller $(20)$ and (21) with the coefficients $c_{1}=100, c_{2}=100, c_{3}=2500, \kappa_{1}=\kappa_{2}=\kappa_{3}=0.25$, together with the learning algorithm (39), (40) and (41) with the coefficients $a_{k}=0.5, \omega_{k}=7.5, a_{\eta}=$ $0.2, \omega_{\eta}=7.4, a_{f_{d}}=1, \omega_{f_{d}}=7.3, q_{1}=q_{2}=500$. For more details about the tuning of the MES coefficients we refer the reader to Ariyur and Krstic (2002), Rotea (2000), Ariyur and Krstić (2003), however, we underline here that the frequencies $\omega_{i}, i=1,2,3$ have been selected high enough to ensure efficient exploration of the search space and ensure convergence and that 

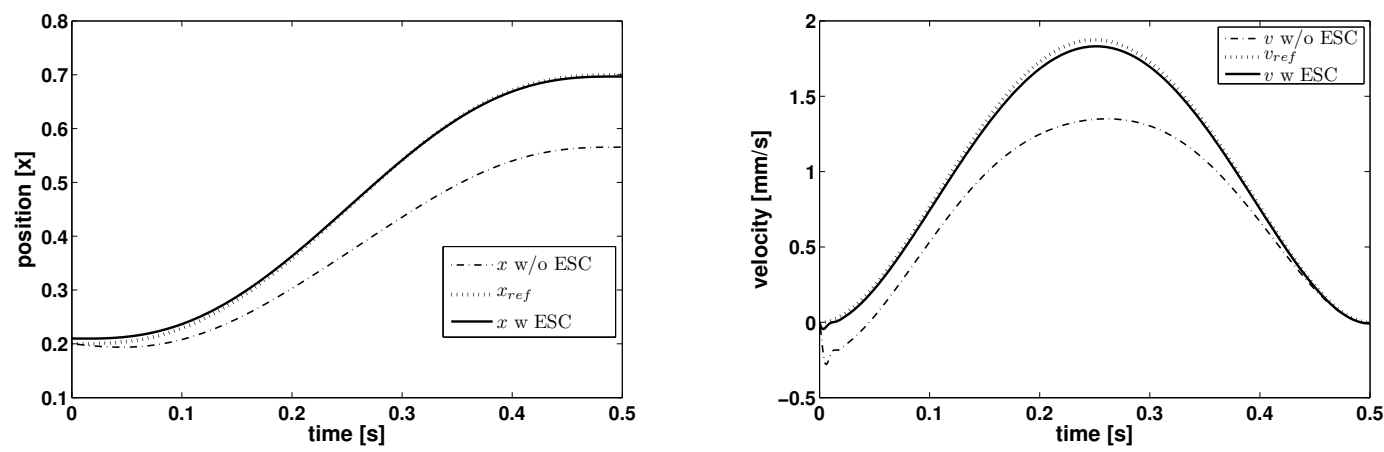

(a) Obtained Armature Position vs. Reference Trajectory

(b) Obtained Armature Velocity vs. Reference Trajectory

Figure 1.: Obtained trajectories vs. Reference Trajectory- Case with uncertain $k, \eta, f_{d}$

the amplitudes $a_{i}$ of the dither signals have been chosen such that the search, i.e., the gradient descent slope in (44), is fast enough for this application. As can be seen in Figures 1(a) and 1(b), the robustification of the backstepping control via extremum seeking greatly improves the tracking performance. Figure 2(a) shows that the cost function decreases below 1 within 20 iterations. It can be seen in Figure 2(b) that the cost starts at an initial value around 9, and decreases rapidly afterwards. Moreover, the estimated parametric uncertainties $\Delta k, \Delta \eta$ and $\Delta f_{d}$ converge to regions around the actual parameter values, as shown on Figure 3. The number of iterations for the estimates to reach the actual value of the parameters may appear to be high. The reason is that the allowed uncertainties in the parameters are large, hence the extremum seeking scheme requires a lot of iterations to improve performance. Furthermore, we purposely tested the challenging case of three simultaneous uncertainties, which makes the space search for the learning algorithm large (note that this case of multiple uncertainties could not be solved with other classical model-based adaptive controllers (Benosman and Atinc (2013)). However, in real-life applications uncertainties accumulate gradually over a long period of time, while the learning algorithm keeps tracking these changes continuously. Thus, the extremum seeking algorithm will be able to improve the controller performance quickly, meaning that it will enhance the backstepping control in fewer iterations. Finally, the control voltage is depicted on Figure 4, which shows an initial high value due to the relatively large simulated initial condition error on the armature position.

Finally, to make the simulation tests closer to a real test-bed validation, we performed the same tests as above, but we introduced more uncertainties on the measured signals and the direct simulation model. We assume that a white noise with a maximum excursion of $0.01 \mathrm{~mm}$ is added to the measured position signal. Indeed, in practical setting the armature position can be measured by precise position sensors, e.g. laser sensors, which can generate noisy signals due to electrical noises or mechanical vibrations of the armature. We also added a random 


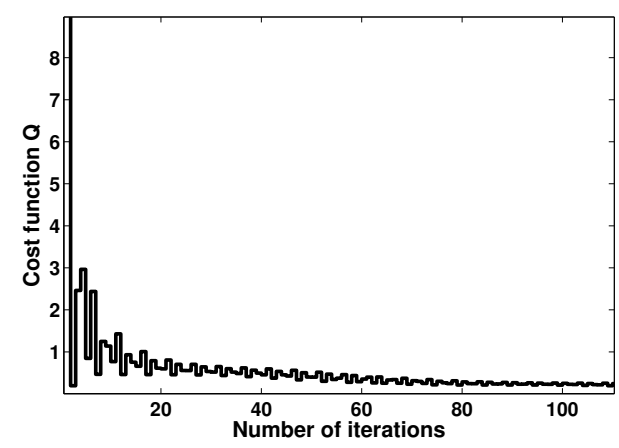

(a) Cost function- zoom

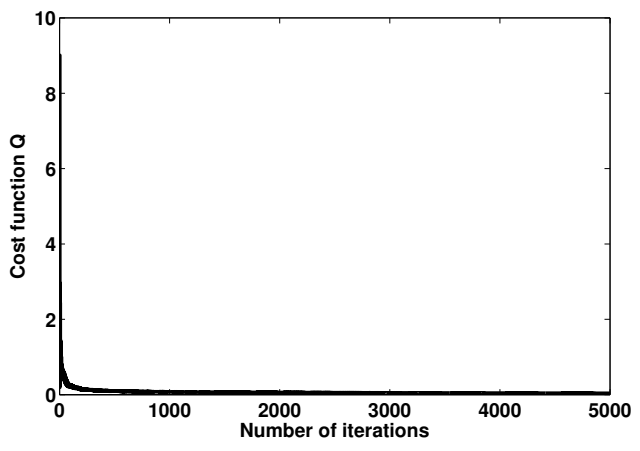

(b) Cost function

Figure 2.: Cost function- Case with uncertain $k, \eta, f_{d}$

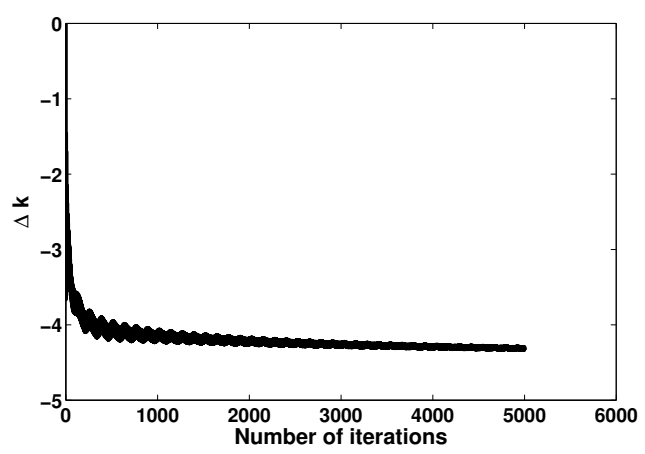

(a) Parameter $k$ estimate

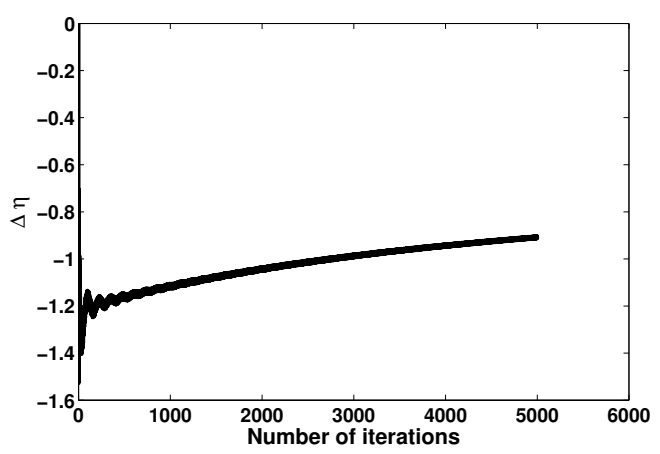

(b) Parameter $\eta$ estimate

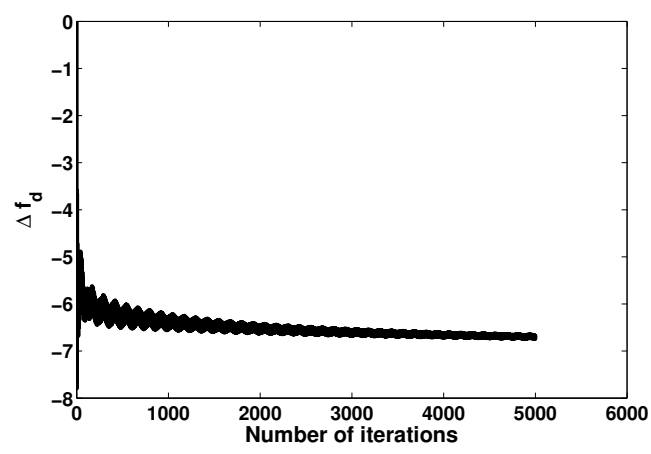

(c) Parameter $f_{d}$ estimate

Figure 3.: Parameters estimates- Case with uncertain $k, \eta, f_{d}$

white noise to the current measurements with an excursion of $2 \times 10^{-6} \mathrm{~A}$. This is a reasonable approximation of the electrical noises in the presently available current sensors, e.g. hall-effect sensors, since these sensors, if properly shielded, have practically very small noise appearing on their output signal. We assumed that the armature velocity is computed from the position signal by direct differentiation. All the measurements are simulated with a sampling rate of $1 \mathrm{~ms}$. Furthermore, we imposed saturations on the voltage signal between 0 and 60 volts. Finally, to 


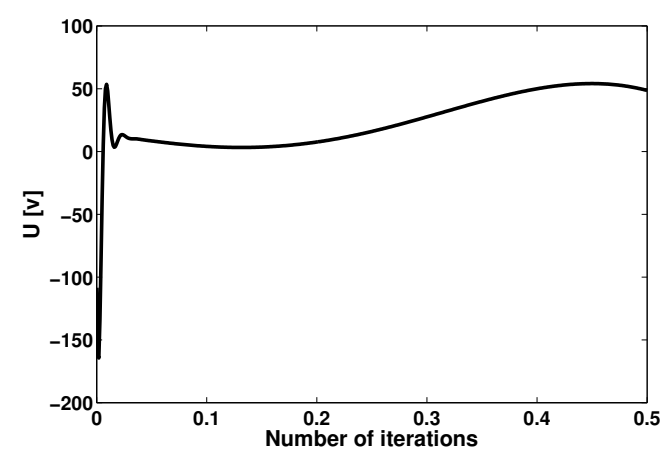

Figure 4.: Control voltage- Case with uncertain $k, \eta, f_{d}$

test the controller performance when dealing with model structural uncertainties, we added in the direct model used in the simulations, the effect of eddy currents on the coil. Following Chladny (2005), eddy current effect was modelled by adding a $R_{e d d y}-L_{e d d y}$ circuit in parallel with the coil's electrical circuit. In this case, the model (9) is modified as follows:

$$
\begin{aligned}
& m \frac{d^{2} x}{d t^{2}}=k\left(x_{0}-x\right)+\eta \frac{d x}{d t}-\frac{a i^{2}}{2(b+x)^{2}}+f_{d} \\
& u=R\left(i_{e d d y}+i\right)+\frac{a}{b+x} \frac{d i}{d t}-\frac{a i}{(b+x)^{2}} \frac{d x}{d t} \\
& \frac{d i_{e d d y}}{d t}=\frac{1}{L_{e d d y}}\left(u-R\left(i+i_{e d d y}\right)-R_{e d d y} i_{e d d y}\right)
\end{aligned}
$$

where, $i_{e d d y}$ denotes eddy current. It was shown in Chladny (2005), via experimental tests, that the model (45) is a good approximation of eddy current effect. We tuned the values of the resistance $R_{e d d y}$ and the inductance $L_{e d d y}$ to have an eddy current maximum amplitude corresponding to $10 \%$ of the coil current $i$ at a nominal functioning of the actuator. The obtained results are shown on Figures 5, 6, 7, and 8, which show a good performance of the proposed controller even in the case of unstructured uncertainties (Eddy-current effect), noisy measurements and input saturation.

\section{Conclusion}

We have studied in this paper the problem of adaptive control for electromagnetic actuators. We have proposed an adaptive controller based on a nonlinear backstepping and a model-free multivariable extremum seeking algorithm. We have proven that the nonlinear backstepping ensures integral input-to-state stability, when considering uncertain parameters appearing linearly in the model. We have also analyzed the stability of the combined backstepping and multi-variable extremum seeking controller, in term of upper-bounds of the tracking error signals. We have shown the performance of the proposed adaptive controller on a numerical example. Future work will include: Applying this approach to other systems and comparing the performance of this 


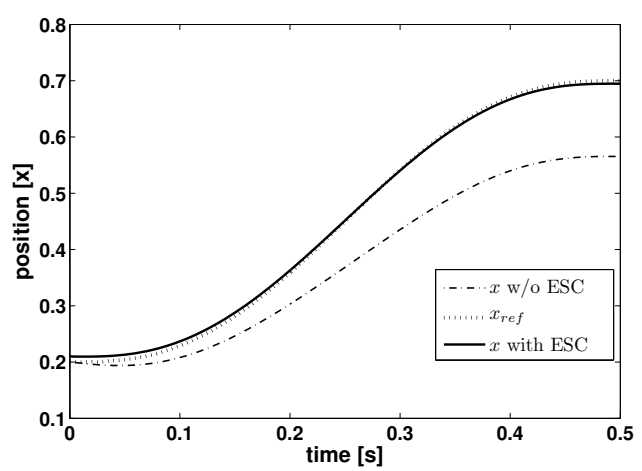

(a) Obtained Armature Position vs. Reference Trajectory

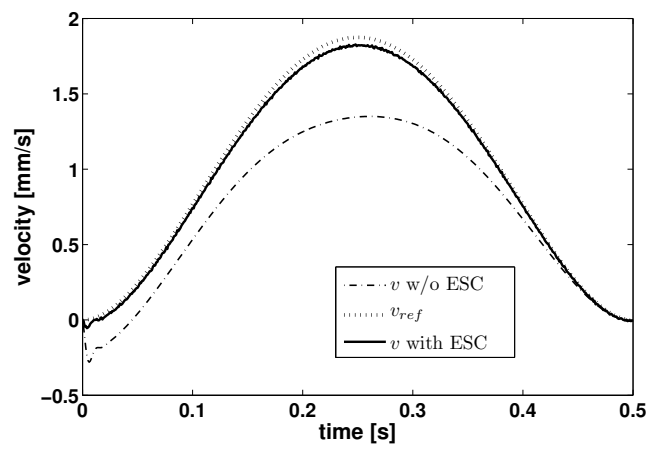

(b) Obtained Armature Velocity vs. Reference Trajectory

Figure 5.: Obtained trajectories vs. Reference Trajectory- Case with uncertain $k, \eta, f_{d^{-}}$Direct model including Eddy-current effect

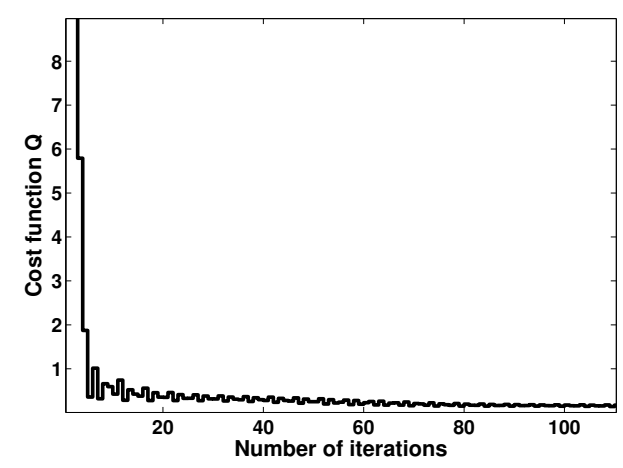

(a) Cost function- zoom

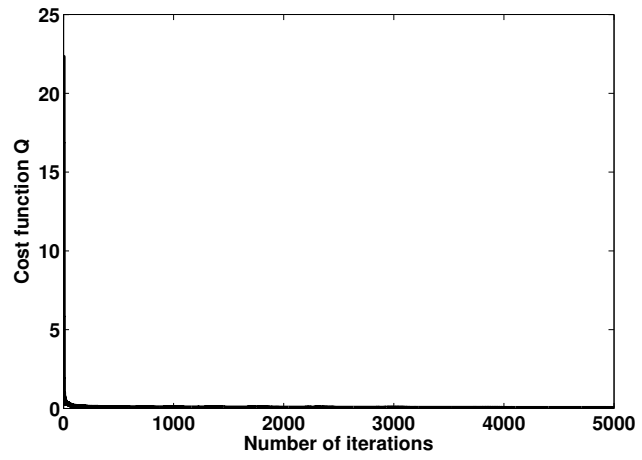

(b) Cost function

Figure 6.: Cost function- Case with uncertain $k, \eta, f_{d^{-}}$Direct model including Eddy-current effect

type of learning-based adaptive controllers to classical adaptive control methods, e.g. Benosman and Atinc (2013), using different MES algorithms with semi-global convergence properties, e.g. Tan (2006), Noase (2011), Scheinker (2013), or other learning paradigms, e.g. reinforcement learning approaches, and comparing the resulting controllers in terms of parameters estimation and tracking performances.

\section{References}

Angeli, D., Sontag, E., and Y., W. (2000), "A characterization of integral input-to-state stability," IEEE Transactions on Automatic Control, 45, 1082-1097.

Ariyur, K.B., and Krstic, M. (2002), "Multivariable extremum seeking feedback: Analysis and design," in Proc. of the Mathematical Theory of Networks and Systems, August, South Bend, 


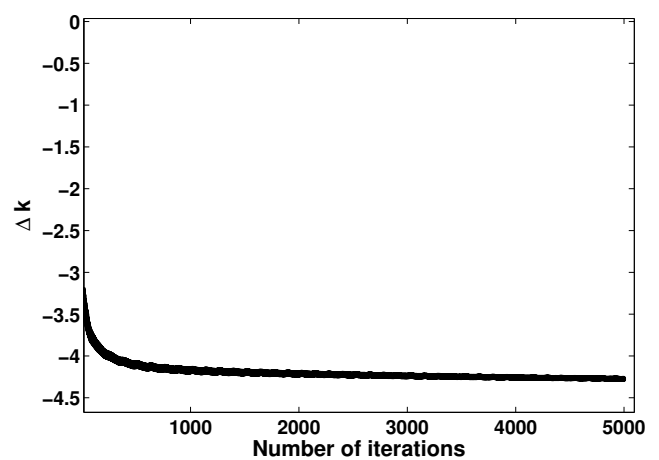

(a) Parameter $k$ estimate

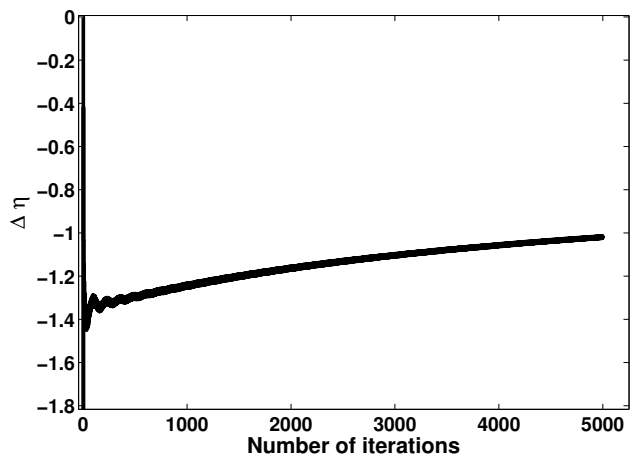

(b) Parameter $\eta$ estimate

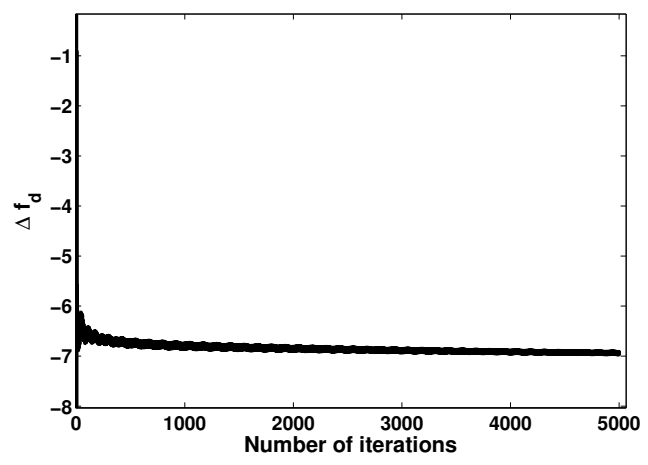

(c) Parameter $f_{d}$ estimate

Figure 7.: Parameters estimates- Case with uncertain $k, \eta, f_{d^{-}}$Direct model including Eddycurrent effect

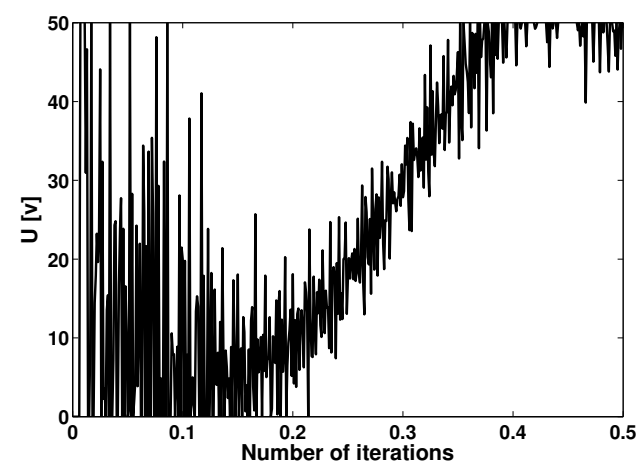

Figure 8.: Control voltage- Case with uncertain $k, \eta, f_{d^{-}}$Direct model including Eddy-current effect

IN.

Ariyur, K., and Krstić, M., Real-time optimization by extremum-seeking control, Wiley-Blackwell (2003).

Atinc, G., and Benosman, M. (2013), "Nonlinear Learning-based Adaptive Control for Electromagnetic Actuators with Proof of Stability," in IEEE, Conference on Decision and Control, 
pp. $1277-1282$.

Benosman, M., and Atinc, G. (2013a), "Multi-Parametric Extremum Seeking-based Learning Control for Electromagnetic Actuators," in American Control Conference, pp. 1914-1919.

Benosman, M., and Atinc, G. (2013), "Nonlinear Adaptive Control of Electromagnetic Actuators," in SIAM Conference on Control and Applications, pp. 29-36.

Benosman, M., and Atinc, G. (2013b), "Nonlinear Learning-based Adaptive Control for Electromagnetic Actuators," in European Control Conference, pp. 2904-2909.

Chladny, R., Koch, C., and Lynch, A. (2005), "Modeling automotive gas-exchange solenoid valve actuators," IEEE Transactions on Magnetics, 41, 1155-1162.

Eyabi, P., and Washington, G. (2006), "Modeling and sensorless control of an electromagnetic valve actuator," Mechatronics, 16, 159-175.

Haddad, W., and Chellaboina, V., Nonlinear dynamical systems and control: a Lyapunov-based approach, Princeton University Press (2008).

Hoffmann, W., Peterson, K., and Stefanopoulou, A. (2003), "Iterative learning control for soft landing of electromechanical valve actuator in camless engines," IEEE, Transactions on Control Systems Technology, 11, 174-184.

Ito, H., and Jiang, Z. (2009), "Necessary and sufficient small gain conditions for integral inputto-state stable systems: A Lyapunov perspective," IEEE Transactions on Automatic Control, $54,2389-2404$.

Kahveci, N., and Kolmanovsky, I. (2010), "Control design for electromagnetic actuators based on backstepping and landing reference governor," in 5th IFAC Symposium on Mechatronic Systems, September, Cambridge, pp. 393-398.

Krstic, M., Kanellakopoulos, I., Kokotovic, P. , Nonlinear and adaptive control design, John Wiley \& Sons New York (1995).

Krstic, M., and Wang, H. (2000), "Stability of extremum seeking feedback for general nonlinear dynamic systems," Automatica, pp. 595-601.

Nesic, D., Nguyen, T., Tan, Y., and Manzie, C. (2013), "A non-gradient approach to global extremum seeking: An adaptation of the Shubert algorithm," Automatica, 49, 809-815.

Noase, W., Tan, Y., Nesic, D., and Manzie, C. (2011), "Non-local stability of a multi-variable extremum-seeking scheme," in IEEE, Australian Control Conference, November, pp. 38-43.

Peterson, K., and Stefanopoulou, A. (2004), "Extremum seeking control for soft landing of electromechanical valve actuator," Automatica, 40, 1063-1069.

Peterson, K., and Stefanopoulou, A. (2003), "Rendering the electromechanical valve actuator globally asymptotically stable," in Proceedings of 42nd IEEE Conference on Decision and Control, December, Maui, HI, pp. 1753-1758. 
Rotea, M.A. (2000), "Analysis of Multivariable Extremum Seeking Algorithms," in American Control Conference, June, pp. 433-437.

Scheinker, A. (2013), "Simultaneous stabilization of and optimization of unkown time-varying systems," in American Control Conference, June, pp. 2643-2648.

Sontag, E., and Wang, Y. (1996), "New characterizations of input-to-state stability," IEEE Transactions on Automatic Control, 41, 1283-1294.

Tai, C., and Tsao, T. (2002), "Control of an electromechanical camless valve actuator," in American Control Conference, May, pp. 262-267.

Tan, Y., Nesic, D., and Mareels, I. (2006), "On non-local stability properties of extremum seeking control," Automatica, pp. 889-903.

Tsai, J., Koch, C., and Saif, M. (2008), "Cycle adaptive feedforward approach control of an electromagnetic valve actuator," in IEEE, Conference on Decision and Control, December, Cancun, Mexico. 